\title{
Multifractality of the quantum Hall wave functions in higher Landau levels
}

\author{
Takamichi Terao and Tsuneyoshi Nakayama \\ Department of Applied Physics, Hokkaido University, Sapporo 060, Japan \\ Hideo Aoki \\ Department of Physics, University of Tokyo, Hongo, Tokyo 113, Japan
}

(March 14, 2018)

\begin{abstract}
To probe the universality class of the quantum Hall system at the metalinsulator critical point, the multifractality of the wave function $\psi$ is studied for higher Landau levels, $N=1,2$, for various range $(\sigma)$ of random potential. We have found that, while the multifractal spectrum $f(\alpha)$ (and consequently the fractal dimension) does vary with $N$, the parabolic form for $f(\alpha)$ indicative of a log-normal distribution of $\psi$ persists in higher Landau levels. If we relate the multifractality with the scaling of localization via the conformal theory, an asymptotic recovery of the single-parameter scaling with increasing $\sigma$ is seen, in agreement with Huckestein's irrelevant scaling field argument.
\end{abstract}

$73.40 \mathrm{Hm}, 64.60 \mathrm{Ak}, 71.30+\mathrm{h}$

Typeset using REVTEX 
It is an intriguing question to ask whether the transition to the Anderson-localized regime in disordered systems is regarded as a phase transition. A suggestion came in 1983 to the effect that the wave function at the Anderson transition should be self-similar (fractal) on all length scales just as the critical point in a phase transition is characterized by a diverging correlation length with the states being scale-invariant 1 To be more precise, the self-similarity in critical wave functions extends beyond a single scale transformation, so the idea has been subsequently developed into the multifractal analysis.

The criticality of the wave function proposed in general has been analyzed for the quantum Hall (QH) system in particular, 1 where the delocalized states, marginally allowed to appear in two dimensions $(d=2)$ in the presence of magnetic fields, coalesce into a single point (the center of each Landau level) on the energy axis. The multifractal analysis has in fact proved to be essential for a full description of the criticality in the $\mathrm{QH}$ system as recently reviewed by Huckestein.

On the other hand, the scaling of localization in $\mathrm{QH}$ systems is still some way from a full understanding: a finite-size scaling study, 5 which looks into the scaling of $\xi_{M} / M=$ $f(M / \xi)$ where $\xi_{M}$ is the localization length in long strips of width $M$, indicates that the single-parameter scaling holds for the $\mathrm{QH}$ system for the Landau index $N=0$, while a two-parameter scaling seems to be required for higher $(N \geq 1)$ ones. In an attempt to reconcile the apparent lack of universality, Huckestein 6 has suggested that, if one introduces an irrelevant scaling length $\xi_{\text {irr }}$ to describe $\xi_{M} / M$ as a function of two variables, $(M / \xi)$ and $\left(M / \xi_{\text {irr }}\right)$, 国 the single-parameter scaling is recovered asymptotically on length scales $>\xi_{\text {irr }}$. The meaning of the second length scale is not fully clarified 7 except that the existence of two parameters is reminiscent of Pruisken's two-parameter scaling that involves the Hall $\left(\sigma_{x y}\right)$ and longitudinal $\left(\sigma_{x x}\right)$ conductivities.

Here natural questions are: (i) how the multifractality will depend on the Landau index, and (ii) can this possibly related to the scaling, which are exactly the purpose of the present paper. It has in fact long been recognized 0 , 8 that the localization length $\xi$ wildly grows with the Landau index $N$, where a mapping onto the nonlinear $\sigma$ model gives an estimate $\xi \sim$ 
$\ell \exp \left(\sigma_{\mathrm{SCBA}}^{2}\right)$ with $\sigma_{\mathrm{SCBA}} \propto(N+1 / 2)$ being $\sigma_{x x}$ in the self-consistent Born approximation 9 and $\ell$ the magnetic length. The $N$ dependence indicates that the scaling has to be discussed on much larger length scales for higher levels, which is indeed one motivation for introducing the irrelevant length. There, we can control the situation if the irrelevant scaling idea is valid: the irrelevant length scale $\xi_{\text {irr }}$ is shown 6 to rapidly decrease when the correlation length of the random potential $\sigma$ is increased from $\ll \ell$ to $\sim \ell$.

Given this background, it will be intriguing how the multifractality will vary with $N$ and/or $\sigma$. We can also propose that the multifractal analysis provides a way to probe the scaling if we combine the multifractality and the scaling. Janßen ${ }^{10}$ has actually suggested that the scaling amplitude $\Lambda_{c}=\lim _{M \rightarrow \infty} \xi_{M} / M$ should be related with the multifractal spectrum $f(\alpha)$ via $\Lambda_{c}=\left[\pi\left(\alpha_{0}-d\right)\right]^{-1}$, where $\alpha_{0}$ is the position of the peak in $f(\alpha)$ against $\alpha$ and $d=2$ is the spatial dimension of the system. This is obtained by relating the typical finite-size scaling variable with the multifractal behavior of the critical wave function through the conformal mapping. 11.2

The Hamiltonian of the QH system is given by

$$
\mathcal{H}=\sum_{N X}|N X\rangle\left(N+\frac{1}{2}\right) \hbar \omega_{c}\left\langle N X\left|+\sum_{N X} \sum_{N^{\prime} X^{\prime}}\right| N X\right\rangle\left\langle N X|V| N^{\prime} X^{\prime}\right\rangle\left\langle N^{\prime} X^{\prime}\right|
$$

with the Landau wave function $|N X\rangle$, the cyclotron frequency $\omega_{c}$, and a random potential $V$. We treat each Landau level separately, assuming that the magnetic field is strong enough with $\left|\left\langle N X|V| N^{\prime} X^{\prime}\right\rangle\right| \ll \hbar \omega_{c}\left(N \neq N^{\prime}\right)$.

Following Ando요영 we can express the random potential as an assembly of scatterers each having a range $d$ as

$$
V(\mathbf{r})=\sum_{i} \frac{V_{i}}{2 \pi \sigma^{2}} e^{-\left|\mathbf{r}-\mathbf{r}_{i}\right|^{2} / 2 \sigma^{2}}
$$

Here the position of the scatterer $\mathbf{r}_{i}$ is randomly distributed, which makes the correlation of the random potential, $\left\langle V(\mathbf{r}) V\left(\mathbf{r}^{\prime}\right)\right\rangle \propto\left(2 \pi \sigma^{2}\right)^{-1} \exp \left(-\left|\mathbf{r}-\mathbf{r}^{\prime}\right|^{2} / 2 \sigma^{2}\right)$, Gaussian as well. The strength, $V_{i}$, of the scatterer is assumed to be $V_{i}= \pm V_{0}$ with an equal probability to make the broadened Landau level symmetric. In the short-range limit, the potential becomes an 
assembly of $\delta$-functions, $V(\mathbf{r})=\sum_{i} V_{i} \delta\left(\mathbf{r}-\mathbf{r}_{i}\right)$. The electronic structure is dominated by the dimensionless concentration of scatterers, $c \equiv 2 \pi \ell^{2} n_{i}=n_{i} / n_{L}$, where $n_{i}$ is the bare concentration and $n_{L}$ the degeneracy (per unit area) of each Landau level.

We consider a QH system of size $L$ under periodic boundary conditions in $x$ and $y$ directions. We take system sizes as large as $L=\left(2 \pi n_{L}\right)^{1 / 2} \ell=200 \ell \sim 300 \ell$, which are considerably larger than those in previous studies.0.13 The Hamiltonian is diagonalized by the Lanczos algorithm with no re-orthogonalization imposed in obtaining the eigenvector. 4 This algorithm enables us to obtain 'inner' eigenvalues and eigenvectors accurately with minimal storage requirements for large-scale eigenvalue problems. The computations of desired eigenvectors have been carried out in two steps. First, the Hamiltonian is tridiagonalized by means of the Lanczos algorithm, and then the tridiagonal matrix is diagonalized in a standard way. Second, the eigenvectors are calculated ¿from the series of reproduced Lanczos vectors and the eigenvectors of the tridiagonal matrix. The size of the tridiagonal matrix is taken to be $3 N_{L} \sim 5 N_{L}$ where $N_{L}$ is the dimension of the Hamiltonian. This achieves an accuracy for normalized eigenvectors $\psi_{\mu}$ better than $\left\|\mathcal{H} \psi_{\mu}-E_{\mu} \psi_{\mu}\right\|=10^{-10}$ even at the band center, where $E_{\mu}$ is the eigenvalue.

Figure 1 depicts a typical spatial behavior of the wave function $|\psi(\mathbf{r})|^{2}$ that has an eigenvalue $E_{\mu}$ closest to the center of $N=0($ a) or $N=1(\mathrm{~b})$ Landau levels for the deltapotentials with the concentration $c=10.0$. Sparsely distributed spikes are conspicuous in the wave functions when shown on a linear scale, which is the characteristic feature at a metal-insulator transition point 1.13 This is particularly so for $N=0$, while the $N \geq 1$ wave function is more homogeneously distributed.

We have then obtained the multifractal spectrum $f(\alpha)$ numerically by the box-counting procedure.131, 5 Namely, we divide the system into $N(\lambda) \equiv 1 / \lambda^{2}$ boxes where $\lambda=\ell / L$ is the ratio of the box size and the system size. The probability $P_{j}(\lambda)$ of finding an electron in the $j$ th box $B_{j}(\lambda)$ is

$$
P_{j}(\lambda) \equiv \int_{B_{j}(\lambda)} d \mathbf{r}|\psi(\mathbf{r})|^{2}
$$


If we define the normalized $q$-th moment of the box probability as

$$
\mu_{j}(\lambda, q) \equiv \frac{\left[P_{j}(\lambda)\right]^{q}}{\sum_{k=1}^{N(\lambda)}\left[P_{k}(\lambda)\right]^{q}},
$$

we can calculate the singularity strength,

$$
\alpha(q)=\lim _{\lambda \rightarrow 0} \frac{\sum_{j=1}^{N(\lambda)} \mu_{j}(\lambda, q) \ln \left[P_{j}(\lambda)\right]}{\ln \lambda},
$$

and the multifractal spectrum,

$$
f(\alpha(q))=\lim _{\lambda \rightarrow 0} \frac{\sum_{j=1}^{N(\lambda)} \mu_{j}(\lambda, q) \ln \left[\mu_{j}(\lambda, q)\right]}{\ln \lambda} .
$$

Equations (6) and (7) yield the multifractal spectrum $f(\alpha)$ in a parameter $(q)$ representation. The $\lim _{\lambda \rightarrow 0}$ is taken by reducing the box size to the smallest physical size, $\ell$, with $\lambda=\ell / L$. The generalized dimension $D(q)$ is derived as $D(q)=[f(\alpha(q))-q \alpha(q)] /(1-q)$. Specifically, $D(2)$ gives the fractal dimension of the wave function, which has been obtained more precisely by the direct determination of the box probabilities.13

Figure 2(a) presents the $f(\alpha)$ spectrum of the critical wave functions at the center of $N=0,1,2$ Landau levels, respectively, where a smooth function is obtained in a finite region of $\alpha$. We have checked that the system size is large enough by confirming that $f(\alpha)$ 's for two different $L=200 \ell, 300 \ell$ agree within statistical errors and that we are indeed looking at the critical wave function.16 If not, the $f(\alpha)$ spectrum would coalesce into a single point $(\alpha, f(\alpha))=(d, d)$ for extended wave functions, or two points, $(\alpha, f(\alpha))=(0,0),(\infty, d)$ for localized ones in the limit $L \rightarrow \infty$.

A salient feature in Fig.2(a) is that (i) reflecting the significant difference seen in Fig.1, the $f(\alpha)$ spectra does vary systematically with $N$, but (ii) for all of $N=0,1,2 f(\alpha)$ fits very well to a parabolic form, 3

$$
f(\alpha)=d-\frac{\left(\alpha-\alpha_{0}\right)^{2}}{4\left(\alpha_{0}-d\right)} .
$$

The fact that the critical wave functions at higher Landau levels as well as the lowest one possess the parabolic form $f(\alpha)$ indicates a log-normal distribution $\exp \left[\left(\ln P-\alpha_{0} \ln \lambda\right)^{2} / 4\left(\alpha_{0}-\right.\right.$ 
d) $\ln \lambda$ ], on the box probability $P$. Since the position of the peak $\alpha_{0}(\alpha$ for $q=0)$, and the curvature are correlated as seen from Eq. (8), $f(\alpha)$ is characterized by a single exponent $\alpha_{0}(N)$, which is a function of $N$. The value of $\alpha_{0}$ decreases from $\alpha_{0}(0)=2.31 \pm 0.02$ to $\alpha_{0}(1)=2.15 \pm 0.02$ and further down to $\alpha_{0}(2)=2.09 \pm 0.01$. This is the first key result of this paper. We can visualize this in a typical profile of the $N=1$ wave function on a logarithmic scale in Fig. 3(b). The figure shows that the fluctuation in the wave fucntion, which is wild on the logarithmic scale, indeed becomes similar to the $N=0$ one (Fig. 3(a)) when a linear transformation on logarithmic scale, $\ln |\psi| \rightarrow\left[\left(\alpha_{0}(0)-d\right) /\left(\alpha_{0}(1)-d\right)\right] \ln |\psi|+$ const, is performed to make the two distributions coincide. The approach of $\alpha_{0}$ to the support of the measure (= spatial dimension $d)$ thus means that the amplitude of wave functions becomes less wildly distributed.

Accordingly, the fractal dimension $D(2)=2 \alpha(2)-f(\alpha(2))$ of the critical wave functions approaches to $d=2$ as we go from $N=0(D(2)=1.50 \pm 0.06)$ to $N=1(D(2)=1.73 \pm$ $0.05)$ and $N=2(D(2)=1.82 \pm 0.05)$, and, consequently, the density autocorrelation, $\left\langle|\psi(\mathbf{r}) \psi(\mathbf{r}+\mathbf{R})|^{2}\right\rangle \sim R^{D(2)-d}$, becomes longer tailed. We have a smaller value for $N=0$ compared with that obtained by the lattice model or the scaling of the static conductivity, 17 but the values of $D(2)$ for $N \geq 1$ are close to those obtained from the exponents of the density-density correlation by Mieck. 18

If we turn to finite-ranged potentials, $\sigma>0$, Figure 2(b) shows the $f(\alpha)$ spectrum for the critical wave function for $N=1$ with $\sigma=0.71 \ell$ and $c=3.0$. The $f(\alpha)$ spectrum continues to be parabolic, but the value of $\alpha_{0}$ significantly increases to $\alpha_{0}=2.32 \pm 0.03$ with decreased $D(2)=1.51 \pm 0.07$. The respective values of $\alpha_{0}$ and $D(2)$ almost go back to those for $N=0$. This is also seen in the profile of the wave function in Fig.1(c). The resemblance to the $N=0$ situation provides a sign of the recovery to the $N=0$ situation.

We can now look at the scaling amplitude estimated as $\Lambda_{c}=\left[\pi\left(\alpha_{0}-d\right)\right]^{-1}$ for $N=1$ as a function of $\beta^{2} \equiv 1+(\sigma / \ell)^{2}$ in Fig. 4. As the correlation length $\sigma$ is increased, the value of $\Lambda_{c}$ approaches to a value that is consistent with an argument by Lee et al.19 on the universal conductivity at the transition with $\Lambda_{c}=1 / \ln (1+\sqrt{2})$. This recovery of the 
single-parameter scaling in the higher Landau level with long-range potential agrees with the finite-size scaling result of Huckesteint.

In conclusion, the multifractal spectrum $f(\alpha)$ at criticality does depend on the Landau inded $N$ while a universality still exists in that its parabolic form is preserved, and the scaling valid for the lowest Landau level is asymptotically recovered as the potential range is increased to $\sim \ell$ for $N=1$. One will be able to see how the localization exponent $\nu$ behaves via the identification $1 / \nu=\alpha_{0}+x \underline{10}$ by calculating the normalization exponent $x$ for higher Landau levels. The $N$ dependence of the fractality will also have an implication on the anomalous diffusion coefficient $D(q, \omega) \sim(q / \sqrt{\omega})^{-\eta}$, where $\eta=d-D(2)$.

One of the authors (H. A.) wishes to thank Yshai Avishai and Hiroshi Imamura for valuable discussions, and Wilhelm Brenig for showing a preprint prior to publication. This work was supported in part by a Grant-in-Aid from the Ministry of Education, Science, and Culture of Japan. Calculations were done on the Supercomputer Center, Institute of Solid State Physics, University of Tokyo. 


\section{REFERENCES}

${ }^{1}$ H. Aoki, J. Phys. C 16, L205 (1983); Phys. Rev. B 33, 7310 (1986).

${ }^{2}$ C. Castellani and L. Peliti, J. Phys. A 19, L429 (1986).

${ }^{3}$ M. Schreiber and H. Grussbach, Phys. Rev. Lett. 67, 607 (1991).

${ }^{4}$ B. Huckestein, Rev. Mod. Phys. 67, 357 (1995).

${ }^{5}$ H. Aoki and T. Ando, Phys. Rev. Lett. 54, 831 (1985); T. Ando and H. Aoki, J. Phys. Soc. Jpn. 54, 2238 (1985).

${ }^{6}$ B. Huckestein, Phys. Rev. Lett. 72, 1080 (1994).

${ }^{7}$ F. Evers and W. Brenig, preprint, attempt to relate the classical-to-quantum localization transition to $\xi_{\text {irr }}$.

8 T. Ando, J. Phys. Soc. Jpn. 53, 3101 (1984).

${ }^{9}$ T. Ando and Y. Uemura, J. Phys. Soc. Jpn. 36, 959 (1974).

${ }^{10}$ M. Janßen, Int. J. Mod. Phys. B 8, 943 (1994).

11 J. L. Cardy, J. Phys. A 17, L385 (1984).

12 J. L. Pichard and G. Sarma, J. Phys. C 14, L617 (1981).

${ }^{13}$ W. Pook and M. Janßen, Z. Phys. B 82, 295 (1991).

${ }^{14}$ J. K. Cullum and R. A. Willoughby, Lanczos algorithms for large symmetric eigenvalue computations (Birkhäuser, Basel, 1985).

${ }^{15}$ A. Chhabra and R. V. Jensen, Phys. Rev. Lett. 62, 1327 (1989).

${ }^{16}$ Y. Avishai, Y. Hatsugai and M. Kohmoto, Phys. Rev. B 51, 13419 (1995).

${ }^{17}$ B.M. Gammel and W. Brenig, Phys. Rev. Lett. 73, 3286 (1994).

${ }^{18}$ B. Mieck, Z. Phys. B 90, 427 (1993). 
${ }^{19}$ D.H. Lee et al, Phys. Rev. Lett. 70, 4130 (1993). 


\section{Figure captions}

FIG. 1. Squared amplitude of a typical wave function on a linear scale at the center of each Landau level for the system of size $300 \ell$ for the $N=0$ Landau level with $\sigma=0$ (top), $N=1$ with $\sigma=0$ (middle), and $N=1$ with $\sigma=0.71 \ell$ (bottom).

FIG. 2. (a) The $f(\alpha)$ spectra (for $|q| \leq 4$ ) of the wave functions at the center of the $N=0,1,2$ Landau levels for short-range random potentials $(\sigma=0)$. The solid circles (squares) represent the system size $200 \ell(300 \ell)$.

(b) The same for a finite-ranged random potential, $\sigma=0.71 \ell\left(\beta^{2}=1.5\right)$, for $N=1$ Landau level.

FIG. 3. Logarithm of the squared amplitude of a typical wave function at the center of $N=0$ Landau level with $\sigma=0$ (top) is compared with the same for $N=1$ with a rescaling of the wave function to make its log-normal distribution the same as for $N=0$ (bottom).

FIG. 4. The scaling amplitude $\Lambda_{c}=\left[\pi\left(\alpha_{0}-d\right)\right]^{-1}$ for $N=1$ against $\beta^{2} \equiv 1+(\sigma / \ell)^{2}$. The horizontal dashed line indicates $\Lambda_{c}=1 / \ln (1+\sqrt{2})$. 